\title{
Beratung vor Regress muss eindeutig sein
}

Nach einem Urteil des Sozialgerichts München muss sich die Beratung des Vertragsarztes nach erstmaliger Überschreitung des Richtliniengrößenvolumens ( $\$ 106$ Abs. 5e S. 1 SGB V) deutlich von der "normalen“ Beratung unterscheiden, die bei jeder Überschreitung der Richtgrößen um mehr als 15\% angezeigt ist ( $\$ 106$ Abs. 5a S. 1 SGB V). An die Beratung erstmalig auffälliger Ärzte nach Abs. 5e sind deutlich höhere Anforderungen zu stellen.

Das Urteil stellt klar, dass dem Vertragsarzt bei erstmaliger Überschrei- tung zumindest eine Beratung durch die Prüfstelle angeboten werden muss. Dieses Angebot kann zwar auch schriftlich erfolgen, aber es reicht nicht, dem Arzt einen Maßnahmenbescheid zu schicken, etwa einen Festsetzungsbescheid zur Erstattung der Mehrkosten oder einen Widerspruchsbescheid. Die Botschaft: So sieht individuelle Beratung nicht aus (Az.: S 28 KA 1344/14).

\section{MMW-KOMMENTAR}

Damit betonen die Richter auch, dass laut § 106 Abs. 5e S. 3 SGB V erst dann eine Erstat- tung festgesetzt werden kann, wenn zuvor eine Beratung angeboten wurde. Dabei muss es sich um eine auf den speziellen Beratungsbedarf des Vertragsarztes ausgerichtete und auf den betroffenen Prüfungszeitraum bezogene Beratung handeln. Eine Ausnahme von diesen Anforderungen gibt es nur, wenn der Vertragsarzt schon seit Jahren sein RichtgröBenvolumen überschreitet und man davon ausgehen kann, dass ihm die Unwirtschaftlichkeit seines Verordnungsumfangs hinlänglich bekannt ist. Dies steht auch im Einklang mit der Rechtsprechung des Bundessozialgerichts zu § 106 Abs. 5a S. 1 SGB V.

\section{Prävention polstert Ihre Honorarreserve auf}

_ Jahrelang wurde darüber diskutiert, im letzten Jahr war es endlich soweit: Das Präventionsgesetz (PrävG) trat in seinen wesentlichen Teilen am 25. Juli 2015 in Kraft. Das Gesetz schafft unter anderem die Voraussetzungen für die Weiterentwicklung der bestehenden Gesundheitsuntersuchung, des Check-up 35. Er soll gemäß $₫ 25$ Abs. 1 SGB V künftig stärker auf die Erfassung und
Bewertung gesundheitlicher Risiken und Belastungen ausgerichtet werden und auch eine präventionsorientierte ärztliche Beratung beinhalten. Auf dieser Grundlage können Ärzte den Patienten individuelle Maßnahmen zur Primärprävention empfehlen. Außerdem sollen künftig die Altersgrenzen, die Häufigkeit des Anspruchs und die anzuwendenden Untersuchungsmethoden
Tab. 1 Vorsorge-Kombination für Erwachsene

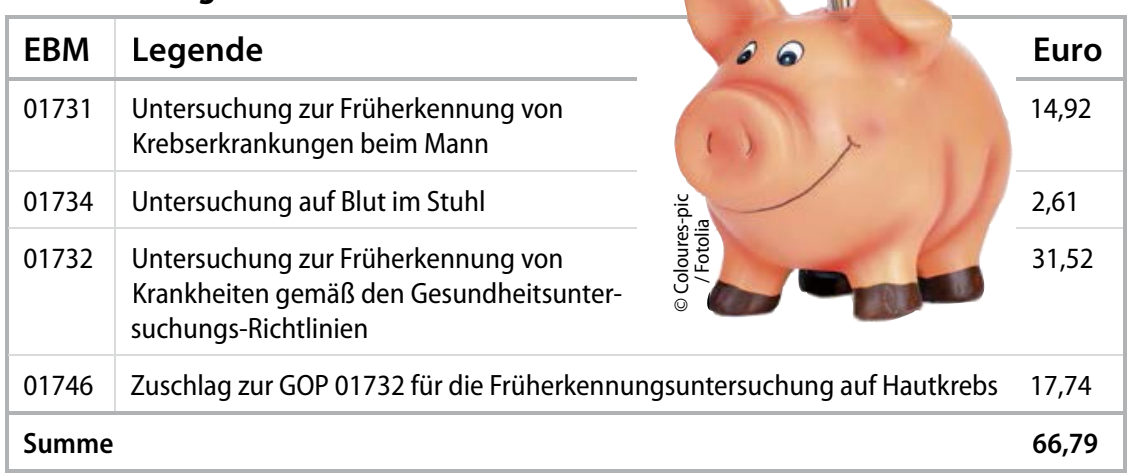

flexibler gehandhabt und besser auf die verschiedenen Zielgruppen ausgerichtet werden.

\section{MMW-KOMMENTAR}

Vorsorgeleistungen in der hausärztlichen Praxis sind auch unter honorarpolitischen Gesichtspunkten zu beachten. Immerhin werden sie extrabudgetär und ohne Mengenbegrenzung zu einem festen Euro-Betrag vergütet. Üblicherweise ist in einer hausärztlichen Praxis davon auszugehen, dass zwischen 60 und $70 \%$ der Patienten ein "vorsorgerelevantes" Alter haben und deshalb für eine Kombinationsuntersuchung aus Krebsvorsorge, Hautkrebsscreening und Check-up infrage kommen (Tab. 1). In einer Praxis mit 1.000 Behandlungsfällen im Quartal ergäbe sich bei einem Potenzial von 60\% der Patienten und einer regelmäßigen Inanspruchnahme alle zwei Jahre ein sicheres Zusatzhonorar von 20.037 Euro pro Jahr! Übrigens sind neben diesen Leistungen auch die Beratung nach Nr. 03230 EBM und ggf. DMP-Untersuchungen berechnungsfähig, was nicht unwichtig ist, da es ja oft parallel einen kurativen Anlass gibt. 